\title{
Lkb1 is a master regulator of VSMC fate and function in mice
}

Zhaohua Cai ${ }^{1,2}$, Ping Song ${ }^{1}$, Shaojin You ${ }^{1}$, Zhixue Liu ${ }^{1}$, Fujie Zhao ${ }^{1}$, Jing Mu ${ }^{1}$, Xiaoxu Zheng ${ }^{1}$, Ye Ding ${ }^{1}$, Lei Xiao ${ }^{3}$, Tharmarajan Ramprasath ${ }^{1}$, Yu Qiu ${ }^{1}$, Ben $\mathrm{He}^{2}$, Ming-Hui Zou ${ }^{1, *}$

${ }^{1}$ Center for Molecular and Translational Medicine, Georgia State University, Atlanta, GA, 30303; ${ }^{2}$ Heart center, Shanghai Chest Hospital, Shanghai Jiaotong University, Shanghai, 200030,

China; ${ }^{3}$ Cardiovascular Research Center, School of Basic Medical Sciences, Xi'an Jiaotong University, Xi'an, 710061, China

10

Short title: Lkb1 regulates VSMC fate and function

\section{*Corresponding Author:}

Ming-Hui Zou, MD, PhD

Center for Molecular and Translational Medicine

Georgia State University

157 Decatur Street SE

Atlanta, Georgia 30303

Phone: 404-413-6637

Fax: 404-413-3580

E-mail:mzou@gsu.edu 


\begin{abstract}
:
Acquisition and maintenance of vascular smooth muscle cell (VSMC) fate are important for vascular development and homeostasis; however, little is known about the key determinant for VSMC fate and vascular homeostasis. We found that VSMC-specific Lkbl ablation in Lkbl flox/flox;Tagln-Cre mice caused severe vascular abnormalities and embryonic lethality. VSMCspecific deletion of $L k b 1$ in tamoxifen-inducible Lkb1 floxflox; Myh11-Cre/ERT2 mice progressively induced aortic/arterial dilation, aneurysm, rupture, and premature death. Single-cell RNA sequencing and imaging-based lineage tracing showed that $L k b 1$-deficient VSMCs underwent dynamic transcriptional reprogramming and transformed gradually from early modulated VSMCs to fibroblast-like, chondrocyte-like, and even osteocyte-like cells. VSMC transformation followed by extracellular matrix remodeling and inflammatory cell infiltration contributed to the arterial aneurysm formation in tamoxifen-induced Lkb1 flox/flox;Myh11-Cre/ERT2 mice. Finally, we found that VSMC-specific $L k b 1$ ablation resulted in decreased vascular contractility, hypotension, and impaired responses to angiotensin II and vessel injury in vivo. $L k b l$ is therefore a key determinant 15 of mouse VSMC fate that prevents VSMC reprogramming and sustains vascular homeostasis. Our findings have important implications for understanding the pathogenesis of aortic aneurysm.
\end{abstract}

Key words: Lkb1, vascular smooth muscle cells, dilation, aneurysm, aortic rupture, cell transformation, cartilaginous metaplasia, scRNA-seq, lineage tracing 
The vascular smooth muscle cell (VSMC) is a differentiated cell type located in the medial layer of vessels. Despite being highly specialized, VSMCs retain remarkable phenotypic plasticity. This plasticity has been frequently associated with VSMC phenotypic switching from a contractile to a synthetic phenotype (1-3). However, VSMCs can also transdifferentiate/transform into other cell types, including macrophage-like, fibroblast-like, osteochondrocyte-like, or mesenchymal stem-cell-like cells (4-8), indicating that this plasticity can extend lineage boundaries. Although emerging evidence strongly implicates the loss of VSMC contractile properties in life-threatening vascular disorders, such as aneurysms, aortic dissection, and even aortic rupture (9-12), it remains unclear whether there is a cause-and-effect relationship between VSMC transformation or changes in VSMC fate and arterial dilation or aneurysm development. Moreover, the master regulator for VSMC fate and plasticity remains largely unknown.

Liver kinase b1 (Lkb1), a tumor suppressor, is mutated and strongly implicated in PeutzJeghers syndrome, cervical carcinoma, and non-small-cell lung carcinomas (13-15). Lkb1 regulates the metabolism of muscle (16), liver (17), pancreas (18), hematopoietic stem cells (19), and $\mathrm{T}_{\text {reg }}$ cells (20). Deletion of $L k b 1$ in mammalian neurons (21), epithelial cells (22), and $\mathrm{T}_{\text {reg }}$ cells (23) disrupts their polarity, differentiation, or lineage identity. Mice deficient in $L k b l$ die at midgestation with vascular abnormalities, mesenchymal cell death, and neural tube defects (24). Deletion of $L k b 1$ in endothelial cells causes endothelial dysfunction and hypertension in mice (25). However, Lkb1 is not known to regulate VSMC fate and vascular homeostasis.

\section{Results}

\section{Cardiovascular defects in smooth-muscle-cell-specific $L k b 1$ deficient mice}

To explore the role of Lkb1 expressed by VSMCs in vessel development and function, we generated mice with smooth muscle cell (SMC)-specific $L k b 1$ deletion ( $L k b 1^{S M K O}$ ) by intercrossing

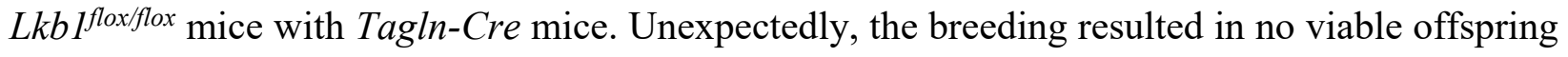
with the Lkbl flox/flox; Tagln-Cre ${ }^{\text {tg }}$ genotype. Genotyping of 11 stillborn offspring identified 8 with

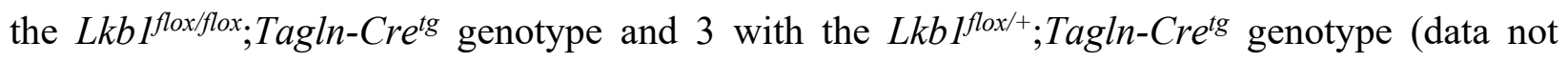
shown). Of 80 embryos dissected between embryonic day (E)8.5 and 20.5, we obtained 26 Lkbl floxfflox; Tagln-Cre ${ }^{\text {tg }}$ embryos which were indeed present at the Mendelian ratio, indicating that $\mathrm{Lkb1}$ is dispensable for implantation (table S1). However, of the $26 \mathrm{Lkbl}^{\text {flox/flox}}$;Tagln-Cre ${ }^{\text {tg }}$ embryos, 21 exhibited visible abnormalities, including small size, developmental delay, absence of normal vessel structures, and other defects (table S1 and fig. S1, A to D). Histological and 
immunofluorescent staining revealed complete absence of vessel structure and VSMC staining in $L k b 1^{S M K O}$ embryos (fig. S1, E and F). These observations suggest that SMC-specific $L k b 1$ deletion resulted in severe vascular abnormalities and embryonic lethality.

Because of the embryonic lethality observed after SMC-specific $L k b 1$ ablation, we generated mice with tamoxifen (TAM)-inducible SMC-specific $L k b 1$ deletion ( $L k b 1^{S M i K O}$ ) by intercrossing Lkb1 floxflox mice with Myh11-Cre/ERT2 mice. In Lkb1 SMiKO mice, Lkb1 was ablated in mature VSMCs after TAM induction (Fig. 1A). Remarkably, all $L k b 1^{S M i K O}$ mice prematurely died within 8 months post-TAM induction, whereas the age-matched wild-type (WT) control mice survived for the duration of the experiment (Fig. 1B). At necropsy, 40 of 42 (95.2\%) Lkbl ${ }^{\text {SMiKO }}$ and 0 of 42 WT control mice developed aortic and/or arterial rupture at an average of $173.0 \pm 5.5$ days postTAM induction (Fig. 1, C and D). The aortic and/or arterial rupture generally occurred in the abdominal aorta (62.5\%), femoral and/or popliteal artery $(40.0 \%)$, and renal artery $(30.0 \%)$ in $\mathrm{Lkbl}^{\text {SMIKO }}$ mice (fig. S2, A to C). Histopathological analysis revealed altered vessel structure and evidence of massive hemorrhage, confirming aortic/arterial rupture (Fig. 1E). When sacrificed beyond 4.5 months post-TAM induction, all $\mathrm{Lkbl}^{\text {SMiKO }}$ mice exhibited aneurysm formation in abdominal aorta, renal artery, iliac artery, femoral artery, and/or popliteal artery (Fig. 1F and fig. $\mathrm{S} 2$, B and C). Over the course of 4.5-month follow-up, both the abdominal and ascending thoracic aortas in $\mathrm{Lkbl}^{\mathrm{SMiKO}}$ mice showed significant and progressive dilation of their internal diameters compared with those in WT control mice (Fig. 1, G to J). Altogether, these data demonstrate that deletion of Lkb1 specifically in mature VSMCs gradually caused aortic/arterial dilation, aneurysm, rupture, and premature death, indicating that Lkb1 plays critical roles in maintaining vascular integrity and homeostasis.

\section{Single-cell RNA sequencing identifies transformed VSMC populations and increased inflammatory cells in $L k b 1^{\text {SMiko }}$ aorta}

To determine the effect of Lkb1 on VSMCs, we performed a time series of droplet-based single-cell RNA sequencing (scRNA-seq) analyses, based on the single aortic cells isolated from WT and $L k b 1^{\text {SMiKO }}$ mice at 1.0, 3.5, and 4.5 months post-TAM induction (fig. S3A). Data from WT and $\mathrm{Lkbl}^{\text {SMIKO }}$ mice were separated into two groups: WT-1M and KO-1M (defined as the early-stage group) and WT-4.5M and KO-3.5M and KO-4.5M (defined as the advanced-stage group). Droplets ( $\mathrm{n}=9202$ ) from early-stage aortas were analyzed, yielding 6928 cells (3154 from WT-1M and 3774 from KO-1M) after rigorous quality control (see the Materials and Methods). 
Graph-based clustering algorithms were used to define 12 distinct clusters, which were further annotated into 5 major cell types by the canonical marker expression: VSMC (clusters 1, 3, 5, 8, expressing VSMC differentiation markers Myh11, Cnn1, and Acta2), early modulated VSMC (clusters 0, 2, 6, 7, 10), fibroblast (cluster 4, expressing Clec3b and Serpinf1), endothelial cell (cluster 9, expressing $V w f$ and Sox 18), and macrophage (cluster 11, expressing Ccl4 and Lyz2) (Fig. 2A and fig. S3, B and C). Clusters 0, 2, 6, 7, and 10 were annotated as early modulated VSMC based on the following characteristics. First, the cells exhibited expression levels of the VSMC differentiation markers consistent with normal VSMCs. Second, the cells displayed high expression levels of Tcap (telethonin) and Rasl10b (RAS-family 10 member B), both of which are highly expressed in cardiac and skeletal muscle; Hapln1 (hyaluronan and proteoglycan link protein 1), an extracellular matrix (ECM) component found in cartilage; and many other genes, including Ifi27l2a, Rbp4, Por, and Egln3. Third, the cells showed slightly upregulated expression of proinflammatory genes, including H2-Ab1, Cd74, Cxcl12, and Alcam (Fig. 2A and fig. S3, B and C). We observed that VSMCs from $L k b 1^{S M i K O}$ mice were exclusively transformed into early modulated VSMCs during this early stage (Fig. 2A).

Next, we investigated single-cell transcriptomes from advanced-stage aortas. We analyzed 7463 droplets in this stage, yielding 5560 cells (1699 from WT-4.5M, 1642 from KO-3.5M, and 2219 from KO-4.5M) after rigorous quality control (see the Materials and Methods), and identified 12 clusters, which were manually curated into 9 different cell types based on distinct marker expression. The most notable change was that the cells in $L k b 1^{S M i K O}$ mice (cells in KO-3.5M and KO-4.5M) shifted from clusters $1,5,7$ to clusters $0,4,2,3$. In other words, cells from clusters 1 , 5 , and 7 were strikingly decreased, whereas cells from clusters $0,4,2$, and 3 were dramatically increased in $\mathrm{Lkbl}^{\mathrm{SMiKO}}$ mice, compared with cells in WT control mice (Fig. 2, B and C). When zooming in on the detailed gene expression profiles of these clusters (Fig. 2D and fig. S4), clusters 1,5 , and 7, which were mainly composed of WT cells showing high-level expression of the VSMC differentiation markers (Myh11, Acta2, and Tagln) but no expression of pro-inflammatory genes (H2-Ab1, H2-Eb1, and Cd74), fibroblast markers (Lum, Fmod, and Lcn2), or chondrocyte markers (Col2al, Col9al, and Chad), suggesting normal VSMCs. Clusters 0 and 4, which were mainly composed of cells from $L k b 1^{S M i K O}$ mice exhibited expression levels of the VSMC differentiation markers consistent with normal VSMCs and moderately upregulated expression of proinflammatory genes, suggesting early transformed VSMCs. Cluster 2, which was mainly 
composed of cells from $L \mathrm{kbl}^{\mathrm{SMiKO}}$ mice, exhibited dramatically downregulated expression of the VSMC differentiation markers and highly upregulated expression of pro-inflammatory genes $(H 2-$ $A b 1, H 2-E b 1, C d 74, C 3, C x c l 12$, and Vcaml) and fibroblast markers, suggesting fibroblast-like cells. Cells in cluster 3 showed exclusively high-level expression of chondrocyte markers and no expression of the VSMC differentiation markers, suggesting chondrocyte-like cells.

Collectively, we found that normal VSMCs dominated the WT aortic cell population (approximately $90 \%$ of aortic cells), but from early to advanced stages, $L k b 1^{S M I K O}$ VSMCs shifted from early modulated VSMCs to fibroblast-like and chondrocyte-like cells (Fig. 2E). At 4.5 months post-TAM induction, $21.8 \%$ and $18.2 \%$ of $L k b 1^{\text {SMiKO }}$ VSMCs had transformed into fibroblast-like and chondrocyte-like cells, respectively, accompanied by $55.4 \%$ of early modulated VSMCs (Fig. 2E). In addition, infiltration of inflammatory cells, particularly macrophages and lymphocytes, was significantly increased in $L k b 1^{S M C i K O}$ aorta. These inflammatory cells constitute up to $10 \%$ of total aortic cells in $L k b 1^{S M i K O}$ aorta at 4.5 months post-TAM induction (Fig. 2, B and C).

To more definitively differentiate distinct transformed VSMC populations, we combined the datasets from the early and advanced stages using robust principal component analysis (RPCA) (see the Materials and Methods). Both normal VSMCs and early modulated VSMCs were clustered together in early and advanced stages; however, fibroblast-like and chondrocyte-like cells were exclusively derived from advanced-stage aorta of $L k b 1^{S M i K O}$ mice (fig. S5, A to C). Taken together, these data suggest that $L k b 1$-deficient mouse VSMCs might undergo a continuous and progressive cell transformation from early modulated VSMCs to fibroblast-like and chondrocyte-like cells. Different Lkbl-deficient VSMCs might stay in different stages of cell transformation.

\section{Single-cell trajectories reveal a gradual cell transformation in $L k b 1^{\text {SMiko }}$ mice}

To further test this hypothesis, we applied the Monocle 2 algorithm to perform cell trajectory analysis using pseudotime reconstitution of four cell groups from the advanced-stage aorta (Fig. 3A). The pseudotime trajectory presented an organized progression of cells from VSMCs to early modulated VSMCs to fibroblast-like and chondrocyte-like cells (Fig. 3, A and B and fig. S6, A and B). Pseudotemporal expression dynamics of specific representative genes also marked the progression of VSMCs to early modulated VSMCs and then fibroblast-like and chondrocyte-like cells (Fig. 3C). VSMC differentiation markers Myh11 and Acta2 showed a gradient of decreasing 
expression along the pseudotime trajectory (Fig. 3D). By contrast, there was a striking and progressive increase in small leucine-rich proteoglycans such as lumican (Lum), fibromodulin (Fmod), and decorin (Dcn) (fig. S6C) and chondrocyte differentiation markers, such as Col2al, Col9a1, Col27a1, Sox9, and osteoprotegerin (Tnfrsf11b) along the pseudotime trajectory from VSMC to chondrocyte-like cell (Fig. 3D and fig. S6C). Similarly, fibroblast markers were upregulated, including dermatopontin ( Dpt), fibulin 1 (Fblnl), and $T g f b i$, predominantly in fibroblast-like cell clusters (Fig. 3D and fig. S6C). Notably, at the transcription level, VSMCs undergoing cell transformation also appeared to be shifting towards a pro-inflammatory phenotype (Fig. 3D and fig. S6C). The results presented here delineate, for the first time, the progressive transformation of mouse VSMCs in an in vivo system.

To obtain molecular insight into the progressive transformation of $\mathrm{Lkbl}^{\text {SMiKO }} \mathrm{VSMCs}$, we further performed differential expression analysis focusing on transcription factors. We identified the top 50 transcription factors showing dynamic expression over the pseudotime (fig. S7). Among them, Klf4 progressively increased along the pseudotime trajectory from VSMC to chondrocytelike cell (fig. S7, A and B). This result is consistent with the well-known role of Klf4 in induction of VSMC transformation $(5,26,27)$. Additionally, the orphan nuclear receptors $\mathrm{Nr} 4 \mathrm{a} 1, \mathrm{Nr} 4 \mathrm{a} 2$, and Nr4a3 strikingly increased over the pseudotime (fig. S7, A and B), suggesting their important roles in regulation of VSMC fate. However, some transcription factors, such as Tgfb1i1, decreased over the pseudotime, (fig. S7, A and B). Our findings reveal that VSMC transformation in Lkb1 $1^{\text {SMiKO }}$ mice was driven by a highly dynamic transcriptional reprogramming.

\section{Visualization of transformed cell populations in $L k b 1^{\text {SMiKO }}$ mice}

We then sought to localize and visualize the transformed VSMCs in aortas or arteries of $\mathrm{Lkbl}^{\text {SMIKO }}$ mice. Therefore, we first checked the thoracic aortic morphology of $L \mathrm{kbl}^{\text {SMiKO }}$ mice at different time points after TAM induction. As early as 1.0-1.5 months after TAM induction, aortas from $L k b 1^{S M i K O}$ mice were dramatically dilated, and the medial wall thickness of the aortas was decreased (Fig. 4, A to C). Consistent with scRNA-seq data, VSMCs from $L k b 1^{\text {SMiKO }}$ mice exhibited nearly identical expression of VSMC differentiation markers ( $\alpha$-actin) as WT VSMCs (Fig. 4B). However, VSMCs from $L k b 1^{S M i K O}$ mice had changed to a flattened, elongated morphology (Fig. 4B) and displayed a corresponding increase in VSMC early-modulation markers and pro-inflammatory genes (fig. S8, A to D), confirming the existence of early-modulated 
VSMCs during this stage. Moreover, unruffled elastic lamellae were observed during this stage (Fig. 4B).

We found that the dilation of the aortas from $L k b 1^{\text {SMiKO }}$ mice had progressively increased (Fig. 4D), which was consistent with echocardiography data (Fig. 1, G to J). Notably, there were some unique cells formed in the aorta of $\mathrm{Lkbl}^{\mathrm{SMiKO}}$ mice at 4.5 months after TAM induction, which exhibited the typical morphology of chondrocytes (Fig. 4E). In addition, we found that $L k b 1$ deletion in VSMCs strongly affected ECM, namely, it led to unruffled elastic lamellae, elastin fiber breakage, and increased collagen deposition (Fig. 4E). The results of the combined Alcian blue-Safranin O staining further revealed the deposition of proteoglycan-rich matrix in $\mathrm{Lkbl}^{\text {SMiKO }}$ aorta (Fig. 4F). These data and scRNA-seq data support that, in addition to VSMC transformation, ECM remodeling contributes to the development of vascular disorders in $\mathrm{Lkbl}^{\text {SMiKO }}$ mice. Our vascular ultrasound analysis showing that $L k b 1^{\text {SMiKO }}$ mice had increased aortic stiffness (fig. S9) further supported ECM remodeling in $\mathrm{Lkbl}^{\mathrm{SMiKO}}$ aorta. However, the Von Kossa staining showed that during this stage (at 4.5 months post-TAM induction) there was no calcification in the thoracic aorta of $L k_{b 1} 1^{S M i K O}$ mice (Fig. 4F), suggesting uncalcified cartilaginous metaplasia. As expected, the uniquely transformed cells were characterized by the lack of expression of VSMC markers $\alpha$ actin and SM-22 $\alpha$ (Fig. 4G) and high-level expression of chondrocyte markers Col2a1 (Fig. 4H) and Sox9 (data not shown), consistent with the scRNA-seq data.

When we followed up mice at 6 months post-TAM induction, we found that the aortic structural alterations in $\mathrm{Lkbl}{ }^{S M i K O}$ mice had become more pronounced. The deposition of collagenrich and proteoglycan-rich matrix extended throughout the entire aortic section (fig. S10, A and B). In addition, there was calcification in the thoracic aorta at this stage, suggesting calcified cartilaginous metaplasia (fig. S10, A and B). Immunofluorescence staining showed that there was no expression of VSMC contraction markers ( $\alpha$-actin and SM-22 $\alpha$ ) anywhere in the entire aorta section (fig. S10C). By contrast, the expression of chondrocyte or fibroblast markers, such as Col2a1, osteopontin (Opn), and lumican (Lum) was markedly increased in Lkbl ${ }^{S M i K O}$ aorta (fig. S10D and fig. S11), suggesting that the transformation of VSMCs in Lkbl ${ }^{\text {SMiKO }}$ mice progressively increased.

We further checked other parts of the aorta, including the aortic root and arch. The aortic root of $L \mathrm{kbl}^{\text {SMiKO }}$ mice exhibited uncalcified cartilaginous metaplasia at 4.5 months post-TAM induction (fig. S12A). However, it progressed to calcified cartilaginous metaplasia at 6.0 months 
post-TAM induction (fig. S12, B and C). For the aortic arch, we found that even at 4.5 months post-TAM induction, calcified cartilaginous metaplasia had formed (fig. S13). Together, these data suggest that different sections of the $L \mathrm{kbl}^{\text {SMiKO }}$ aorta might have different rates and patterns of VSMC transformation progression.

Because $L k b 1$ deletion occurs in all vessels that contain VSMCs, we sought to determine the effect of $L k b 1$ on some medium-sized arteries (muscular arteries). As early as 1.0-1.5 months after TAM induction, the femoral arteries were dramatically dilated (fig. S14A). With the passage of time, non-ruptured and ruptured femoral artery aneurysms were found in $\mathrm{Lkbl}^{S M i K O}$ mice (fig. S14B). Most importantly, we found that the VSMCs were transformed into fibroblast-like cells that were Dpt-positive and Fbln1-positive (fig. S14C). A similar phenomenon was observed in the renal arteries (data not shown). Therefore, we conclude that loss of $L k b l$ results in the transformation of VSMCs to other cell types, such as fibroblast-like or chondrocyte-like cells. However, different types of arteries or different sections of the artery or aorta might have distinct types of cell transformation.

Lineage tracing of transformed cell populations in $L k b 1^{\text {SMiKo }}$ mice

To demonstrate the direct transformation of VSMCs into fibroblast-like and chondrocyte-like cells in $L k b 1^{S M i K O}$ mice, we induced SMC lineage tracing by administrating TAM to triple-

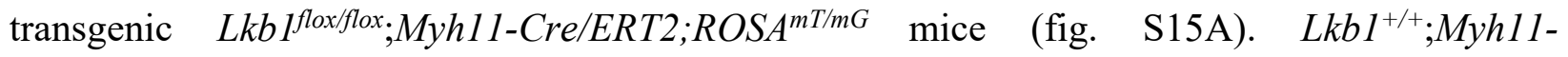
Cre/ERT2;ROSA ${ }^{m T / m G}$ mice were used as controls. tdTomato signals in the vessels were mainly 20 found in the endothelial cells, whereas GFP signals were found in the VSMCs of different kinds of arteries, including aorta, aortic root, femoral artery, renal artery, and coronary artery (fig. S15B). Note that $\mathrm{GFP}^{+}$VSMCs exhibited normal expression of VSMC differentiation marker $\alpha$-actin throughout the entire thoracic aorta of control mice (Fig. 5A). However, immunostaining revealed a progressive decrease of $\alpha$-actin expression in $\mathrm{GFP}^{+} \mathrm{VSMCs}$ in the thoracic aorta of Lkbl flox/flox;Myh11-Cre/ERT2;ROSA ${ }^{m T / m G}$ mice compared with control mice, which is accompanied by a progressive increase of the proteoglycan-rich matrix deposition (Fig. 5A). More importantly, in thoracic aorta, abdominal aorta, and aortic arch, medial $\mathrm{GFP}^{+}$cells that did not express VSMC differentiation marker $\alpha$-actin had acquired characteristics of chondrocytes (Fig. 5, A and B, and fig. S16). These data strongly suggest that $L k b 1^{S M i K O}$ VSMCs directly and gradually transformed into chondrocyte-like cells. 
In addition, we also found that in Lkbl flox/flox;Myh11-Cre/ERT2;ROSA $A^{m T / m G}$ mice that developed femoral and renal artery aneurysms, the $\mathrm{GFP}^{+}$cells were characterized by lack of expression of VSMC marker $\alpha$-actin (fig. S17). Therefore, these data highlighted the important role of progressive VSMC transformation in the pathogenesis of arterial aneurysm.

\section{Impaired vascular function in $L k b 1^{\text {SMiKO }}$ mice}

Given the marked effect of Lkb1 on mouse VSMC fate and vessel morphology changes, we sought to examine the role of Lkb1 in VSMC and arterial function. We first performed blood pressure measurement in WT and $\mathrm{Lkbl}^{\mathrm{SMIKO}}$ mice and found that $\mathrm{Lkb1^{SMiKO }}$ mice displayed low blood pressure compared to WT control mice, including systolic, diastolic, and mean blood pressure (Fig. 6A). $\mathrm{Lkbl}^{\text {SMiKO }}$ mice also showed dramatically decreased vascular smooth muscle contractility induced by U46619 and phenylephrine (PE), as assessed by myographic testing of isolated mesenteric arterial rings (Fig. 6, B to D).

We further tested the effect of VSMC-specific $L k b l$ ablation on angiotensin II (AngII)induced hypertension. AngII treatment induced a strong increase in blood pressure in WT control mice, whereas this effect was much more limited in $\mathrm{Lbbl}^{\text {SMiKO }}$ mice (Fig. 6E). Interestingly, $\mathrm{Lkbl}^{\text {SMIKO }}$ mice had larger internal aortic diameters than those of WT control mice with or without AngII treatment (Fig. 6F). However, VSMC-specific Lkbl ablation abolished Ang II-induced aortic medial thickening (Fig. 6F). In addition, AngII-induced cardiac hypertrophy was also less severe in $\mathrm{Lkbl}^{\mathrm{SMiKO}}$ mice than in WT control mice (fig. S18). Together, these data suggest that Lkb1-deficient VSMCs exhibited a defective response to AngII in vivo.

We further checked the role of Lkb1 in the response to injury of the blood vessel wall, employing the left common carotid artery-ligation mouse model. WT arteries showed obvious neointima formation and wall thickening after carotid ligation. Remarkably, the $L k b 1^{S M i K O}$ arteries developed a severe, organized thrombus that occupied an abnormally enlarged lumen (fig. S19, A and B). Elastic laminae in $\mathrm{Lkbl}^{\text {SMiKO }}$ vessels were distended, as indicated by the absence of undulations, and frequently disrupted (fig. S19B). Comparing the medial wall thickness between WT and $L k b 1^{S M C I K O}$ mice revealed that the media was extremely thin in $L k b 1^{S M i K O}$ vessels after injury (fig. S19, A to C). Immunofluorescence staining showed the lack of expression of VSMC contraction markers ( $\alpha$-actin) throughout the whole $L k b 1^{S M i K O}$ vessel section after injury (fig. 30 S19D). These data indicate that $L k b 1^{\text {SMCiKO }}$ vessels were unable to undergo injury-induced neointima formation. 
Taken together, these data suggest that Lkb1 deficiency in VSMCs results in hypotension, decreased vascular contractility, and impaired responses to AngII and vessel injury in mice in vivo. Because mice used for AngII infusion and carotid artery ligation experiments were 2 weeks postTAM induction, these data further established that transformed VSMCs, even early modulated VSMCs, would lose the physiological and pathological functions of VSMCs.

\section{Discussion}

Although two decades have passed since the discovery that $\mathrm{Lkbl}^{-/-}$mice die at mid-gestation with vascular abnormalities, mesenchymal cell death, and neural tube defects (24), the role of Lkb1 in VSMC fate and vascular homeostasis remains largely unknown. In this study, we generated two VSMC-specific (Tagln-Cre and Myh11-Cre/ERT2 transgenic) Lkb1-deficient mouse models and found that $\mathrm{Lkb1}$ is required for acquisition and maintenance of VSMC fate. We demonstrated that: (1) VSMC-specific Lkbl deletion by SM22 $\alpha$-cre leads to severe vascular abnormalities and embryonic lethality. (2) After targeted Lkbl ablation by TAM-inducible Myh11-Cre/ERT2, mature VSMCs undergo transcriptional reprogramming and transform gradually from early modulated VSMCs to fibroblast-like, chondrocyte-like (cartilage), and even osteocyte-like (calcification) cells, leading to aortic/arterial dilation, aneurysm, and rupture.

Because the phenomenon of VSMC transdifferentiation/transformation has been primarily studied by stimulating cultured VSMCs with lipids and various growth factors (28-30) or using mouse models of atherosclerosis $(8,31-34)$, here we provide a unique in vivo mouse model of spontaneous VSMC transformation. Most importantly, by combining scRNA-seq, lineage tracing, and histological analysis, we clearly displayed several distinct transformed cell types or states that occur at different stages of VSMC transformation. We found that VSMCs that undergo cellular transformation appear to exhibit a continuous trajectory from early modulated VSMCs to fibroblast-like and chondrocyte-like cells, which is driven by a highly dynamic transcriptional reprogramming. In particular, we defined the early modulated VSMC as an intermediate cell state during VSMC transformation for the first time. Moreover, we found that the $L k b 1$ deletion-induced transformed VSMCs would lose the physiological and pathological functions of VSMCs, even in early stage as early modulated VSMCs. Therefore, our work provides a valuable mouse model to investigate the continuous and progressive process of VSMC transformation. 
We also found that $\mathrm{Lkbl}^{\text {SMiKO }}$ mice spontaneously developed arterial aneurysms in abdominal aorta, renal artery, iliac artery, femoral artery, and/or popliteal artery, which is highly reminiscent of human aneurysms (35). Therefore, we also provide an ideal animal model to capture the trajectory of arterial aneurysm formation that resembles human anatomy and pathophysiology. Moreover, our results shed new light on the role of VSMC transformation in arterial aneurysm and suggest that progressive VSMC transformation is the driving force for arterial dilation and aneurysm formation.

Our findings suggest that, in addition to VSMC transformation, ECM remodeling plays important roles in vascular disorders. We found that Lkb1 deficiency in VSMCs led to striking ECM remodeling. In $L k b 1^{S M i K O}$ aorta, the elastic lamellae become unruffled, fragmented, and discontinuous. Most importantly, $\mathrm{Lkbl}^{\text {SMiKO }}$ aorta exhibited excessive deposition of collagen-rich and proteoglycan-rich matrix, accompanied by increased aortic stiffness. These observations provide clear evidence supporting the notion that segmental aortic stiffening is an early pathomechanism for aneurysm progression (36). Further, we also provided data showing the increase in inflammatory cell infiltration during this process. Taking these data together, we speculate that VSMC transformation promotes ECM remodeling and inflammatory cell infiltration, which may further accelerate the transformation of VSMCs, leading to aneurysm formation.

In conclusion, the present study introduces the novel concept that VSMC transformation followed by ECM remodeling and inflammatory cell infiltration contributes to aortic or arterial aneurysm formation, and that $\mathrm{Lkbl}$ is the predominant regulator of mouse VSMC fate maintenance. In other words, aneurysm progression can be regarded as a complex biological process involving VSMC-transformation-driven remodeling of the vessel wall. An improved understanding of VSMC fate regulation may ultimately lead to new approaches to prevent and treat arterial dilation and aneurysm. 


\section{References and Notes:}

1. J. A. Beamish, P. He, K. Kottke-Marchant, R. E. Marchant, Molecular regulation of contractile smooth muscle cell phenotype: implications for vascular tissue engineering. Tissue Eng Part B Rev 16, 467-491 (2010).

2. M. R. Alexander, G. K. Owens, Epigenetic control of smooth muscle cell differentiation and phenotypic switching in vascular development and disease. Annu Rev Physiol 74, 13-40 (2012).

3. P. Petsophonsakul et al., Role of Vascular Smooth Muscle Cell Phenotypic Switching and Calcification in Aortic Aneurysm Formation. Arterioscler Thromb Vasc Biol 39, 1351-1368 (2019).

4. S. Feil et al., Transdifferentiation of vascular smooth muscle cells to macrophage-like cells during atherogenesis. Circ Res 115, 662-667 (2014).

5. L. S. Shankman et al., KLF4-dependent phenotypic modulation of smooth muscle cells has a key role in atherosclerotic plaque pathogenesis (vol 21, pg 628, 2015). Nat Med 22, 217-217 (2016).

6. M. Y. Speer et al., Smooth muscle cells give rise to osteochondrogenic precursors and chondrocytes in calcifying arteries. Circ Res 104, 733-741 (2009).

7. M. W. Majesky et al., Differentiated Smooth Muscle Cells Generate a Subpopulation of Resident Vascular Progenitor Cells in the Adventitia Regulated by Klf4. Circulation Research 120, 296-+ (2017).

8. R. C. Wirka et al., Atheroprotective roles of smooth muscle cell phenotypic modulation and the TCF21 disease gene as revealed by single-cell analysis. Nat Med 25, 1280-1289 (2019).

9. G. Ailawadi et al., Smooth muscle phenotypic modulation is an early event in aortic aneurysms. $J$ Thorac Cardiov Sur 138, 1392-1399 (2009).

10. D. C. Guo et al., Mutations in smooth muscle alpha-actin (ACTA2) lead to thoracic aortic aneurysms and dissections. Nat Genet 39, 1488-1493 (2007).

11. L. Zhu et al., Mutations in myosin heavy chain 11 cause a syndrome associating thoracic aortic aneurysm/aortic dissection and patent ductus arteriosus. Nat Genet 38, 343-349 (2006).

12. M. E. Lindsay, H. C. Dietz, Lessons on the pathogenesis of aneurysm from heritable conditions. Nature 473, 308-316 (2011).

13. A. Hemminki et al., A serine/threonine kinase gene defective in Peutz-Jeghers syndrome. Nature 391, 184187 (1998).

14. N. Bardeesy et al., Loss of the Lkb1 tumour suppressor provokes intestinal polyposis but resistance to transformation. Nature 419, 162-167 (2002).

15. H. Ji et al., LKB1 modulates lung cancer differentiation and metastasis. Nature 448, 807-810 (2007).

16. K. Sakamoto et al., Deficiency of LKB1 in skeletal muscle prevents AMPK activation and glucose uptake during contraction. Embo J 24, 1810-1820 (2005).

17. R. J. Shaw et al., The kinase LKB1 mediates glucose homeostasis in liver and therapeutic effects of metformin. Science 310, 1642-1646 (2005).

18. A. Fu et al., Loss of Lkb1 in adult beta cells increases beta cell mass and enhances glucose tolerance in mice. Cell Metab 10, 285-295 (2009).

19. D. Nakada, T. L. Saunders, S. J. Morrison, Lkb1 regulates cell cycle and energy metabolism in haematopoietic stem cells. Nature 468, 653-658 (2010).

20. K. Yang et al., Homeostatic control of metabolic and functional fitness of Treg cells by LKB1 signalling. Nature 548, 602-606 (2017).

21. A. P. Barnes et al., LKB1 and SAD kinases define a pathway required for the polarization of cortical neurons. Cell 129, 549-563 (2007).

22. B. Y. Shorning et al., Lkb1 deficiency alters goblet and paneth cell differentiation in the small intestine. PLoS One 4, e4264 (2009).

23. D. Wu et al., Lkb1 maintains Treg cell lineage identity. Nat Commun 8, 15876 (2017).

24. A. Ylikorkala et al., Vascular abnormalities and deregulation of VEGF in Lkb1-deficient mice. Science 293, 1323-1326 (2001).

25. W. Zhang et al., Endothelial cell-specific liver kinase B1 deletion causes endothelial dysfunction and hypertension in mice in vivo. Circulation 129, 1428-1439 (2014).

26. T. Yoshida, M. Yamashita, M. Hayashi, Kruppel-like factor 4 contributes to high phosphate-induced phenotypic switching of vascular smooth muscle cells into osteogenic cells. $J$ Biol Chem 287, 25706-25714 (2012). 
27. P. Y. Chen et al., Smooth Muscle Cell Reprogramming in Aortic Aneurysms. Cell Stem Cell 26, 542-557 e511 (2020).

28. N. Clement et al., Notch3 and IL-1 beta exert opposing effects on a vascular smooth muscle cell inflammatory pathway in which NF-kappa B drives crosstalk. J Cell Sci 120, 3352-3361 (2007).

29. N. A. Pidkovka et al., Oxidized phospholipids induce phenotypic switching of vascular smooth muscle cells in vivo and in vitro. Circ Res 101, 792-801 (2007).

30. J. X. Rong, M. Shapiro, E. Trogan, E. A. Fisher, Transdifferentiation of mouse aortic smooth muscle cells to a macrophage-like state after cholesterol loading. Proc Natl Acad Sci U S A 100, 13531-13536 (2003).

31. M. Rattazzi et al., Calcification of advanced atherosclerotic lesions in the innominate arteries of ApoEdeficient mice: potential role of chondrocyte-like cells. Arterioscler Thromb Vasc Biol 25, 1420-1425 (2005).

32. Y. V. Bobryshev, Transdifferentiation of smooth muscle cells into chondrocytes in atherosclerotic arteries in situ: implications for diffuse intimal calcification. J Pathol 205, 641-650 (2005).

33. B. J. Bennett et al., Osteoprotegerin inactivation accelerates advanced atherosclerotic lesion progression and calcification in older ApoE-/- mice. Arterioscler Thromb Vasc Biol 26, 2117-2124 (2006).

15 34. P. Y. Chen et al., Smooth Muscle Cell Reprogramming in Aortic Aneurysms. Cell Stem Cell 26, 542-+ (2020).

35. P. E. Norman, J. T. Powell, Site specificity of aneurysmal disease. Circulation 121, 560-568 (2010).

36. U. Raaz et al., Segmental aortic stiffening contributes to experimental abdominal aortic aneurysm development. Circulation 131, 1783-1795 (2015). 
Acknowledgments: This study was supported by the National Institutes of Health grants (HL079584, HL080499, HL089920, HL110488, HL128014, HL132500, HL137371, HL140954, HL142287, AG047776, and CA213022). This work was, in part, supported by the Georgia Research Alliance. M.-H.Z. is a Georgia Research Alliance Eminent Scholar in Molecular Medicine. Author contributions: Z.C. designed and executed the experiments, analyzed the data and wrote the manuscript. P.S., S.Y., and Z.L. provided advice and analyzed the data. F.Z., J.M., X.Z., Y.D., L.X., T.R., and Y.Q. provided technical assistance and advice. B. H. provided advice, analyzed the data and revised the manuscript. M.-H.Z. conceived and designed the experiments, analyzed the data, and revised the manuscript. Competing interests: The authors declare no competing interests.

\section{Supplementary Materials:}

Materials and Methods

Figures S1-S19

15 Tables S1-S2

References (37-47) 
A

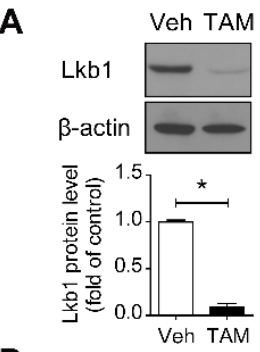

D

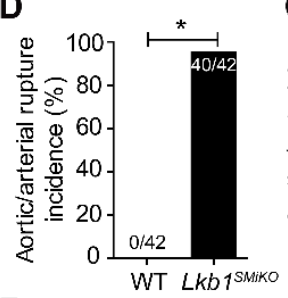

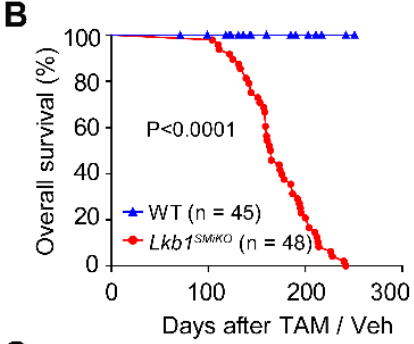

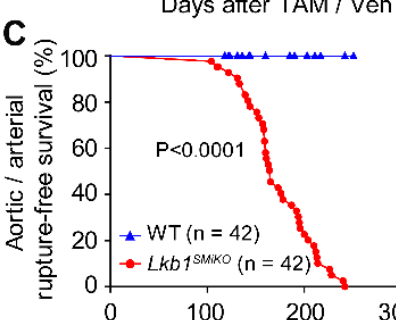

E

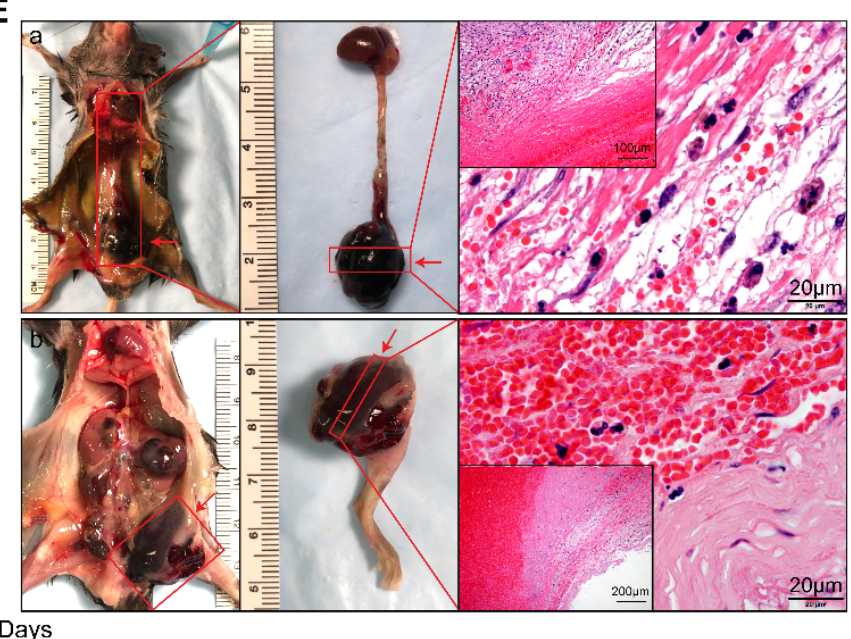

$\mathbf{F}$

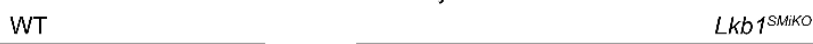
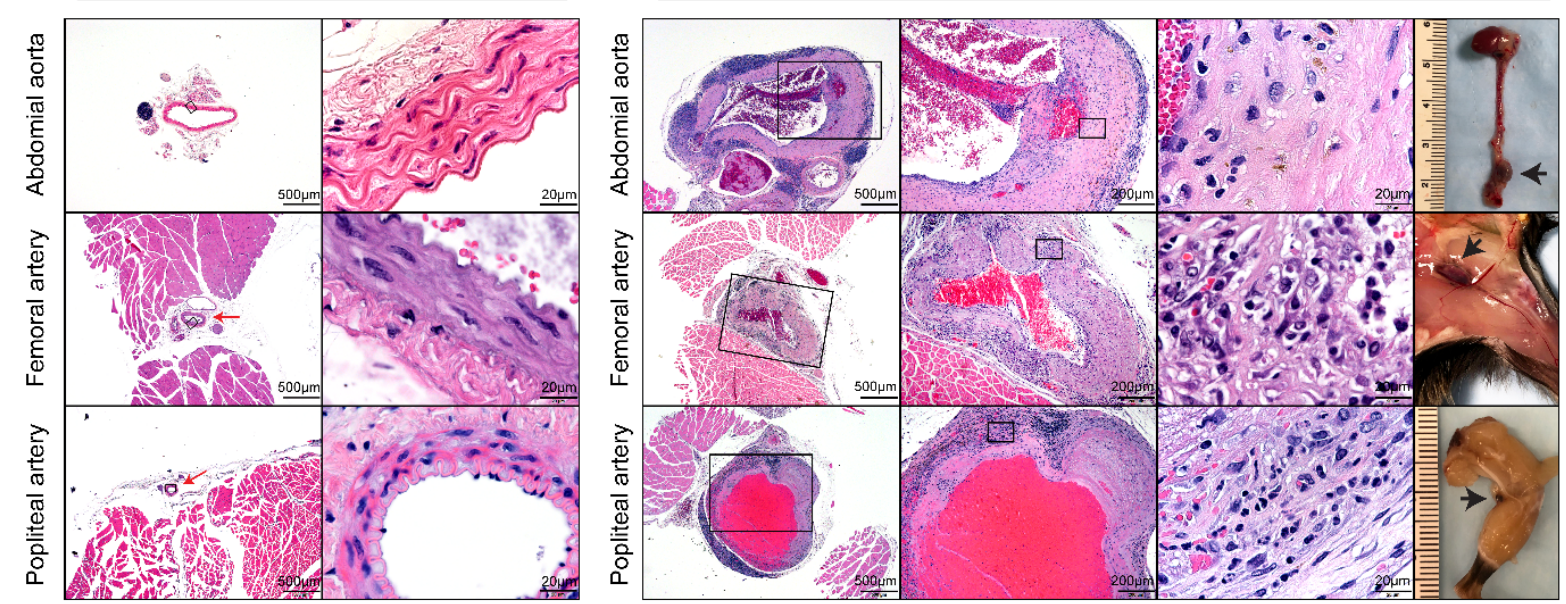

G
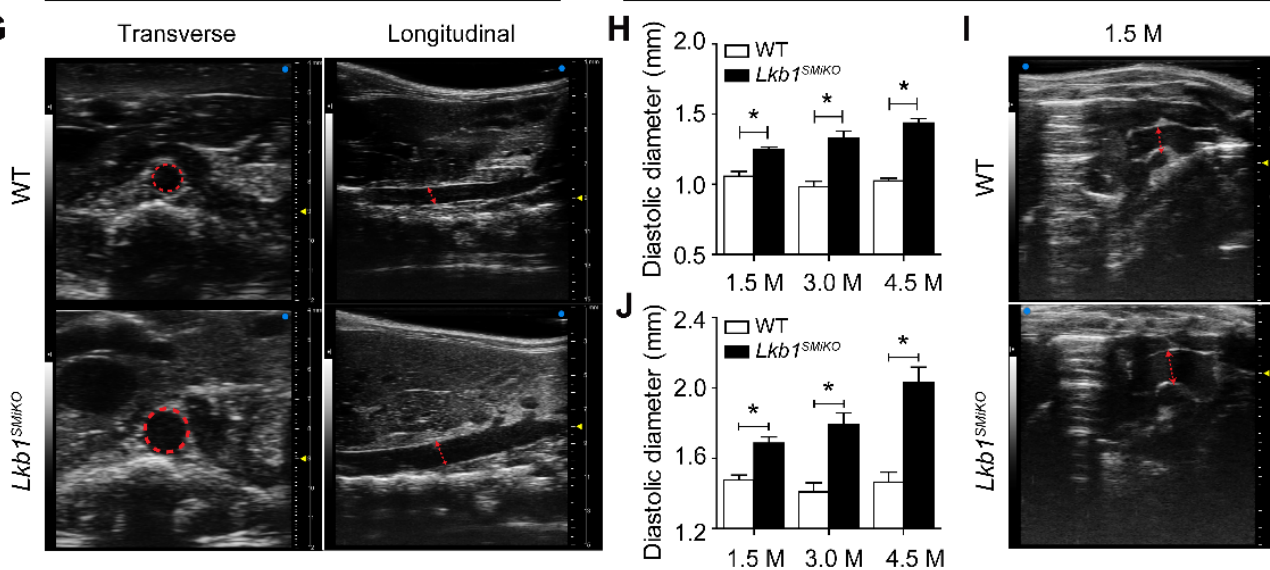

$4.5 \mathrm{M}$

Fig.1. Deletion of $\boldsymbol{L k b 1}$ in mature VSMCs causes aortic or arterial rupture and premature

death. (A) Analysis of Lkb1 expression in primary VSMCs isolated from the aortas of Lkbl floxflox;Myh11-Cre/ERT2 mice treated with tamoxifen (TAM) or vehicle (Veh). (B and C)

Overall survival and aortic/arterial rupture-free survival of wild-type (WT) and $L \mathrm{~Kb}^{\text {SMiKO }}{ }^{\text {mice. }}$

Statistical analysis was performed using the log-rank test. (D) Aortic/arterial rupture incidence in 
WT and $L k b 1^{S M I K O}$ mice. Statistics were determined using Fisher's exact test. (E) Macroscopic images and hematoxylin-and-eosin (H\&E) staining illustrating ruptured vessels in $\mathrm{Lkbl}^{\text {SMiKO }}$ mice. Red arrows denote ruptured aorta (a) or femoral artery (b). (F) Macroscopic images and H\&E staining of abdominal aorta, femoral artery, and popliteal artery in WT and $L k b 1^{\text {SMiKO }}$ mice. Boxes indicate fields shown at higher magnification in images to the immediate right. Red arrows denote the arteries. Black arrows denote aneurysms. (G-J) Representative images and quantification of the abdominal ( $\mathrm{G}$ and $\mathrm{H}$ ) and ascending thoracic ( $\mathrm{I}$ and $\mathrm{J}$ ) aortas monitored by ultrasound from WT and $L k b 1^{\text {SMiKO }}$ mice. Red dashed circles and arrows indicate aortic internal diameter. 
A
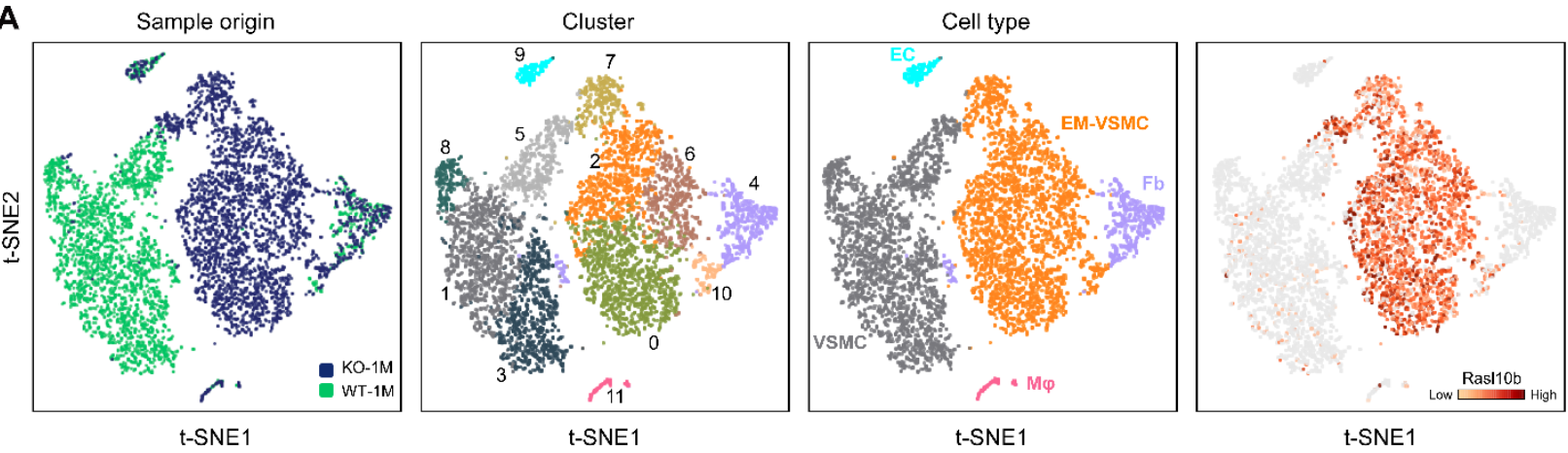

B
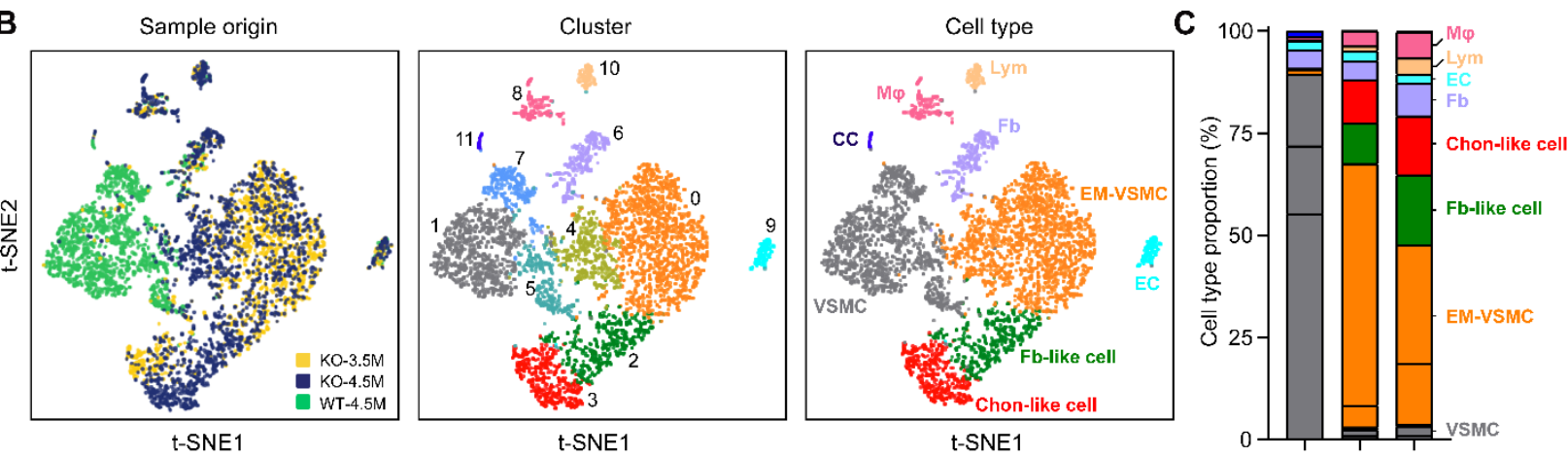

D
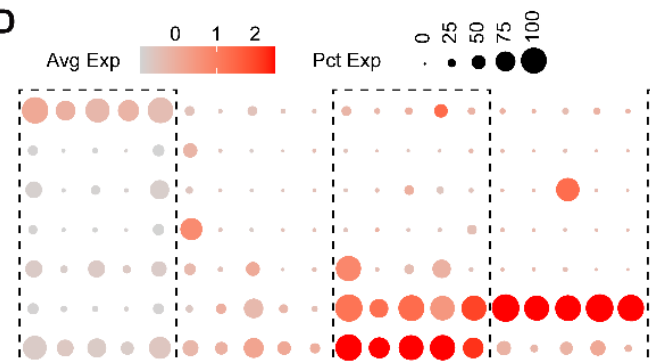

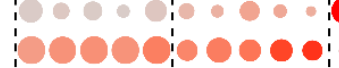

100000000

10000

10000

10000

t-SNE1

t-SNE1
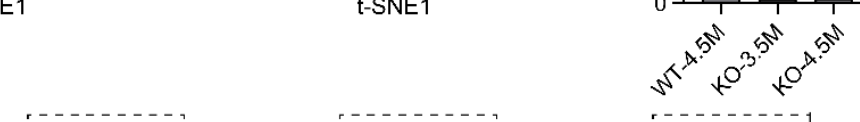

$11 \mathrm{CC}$
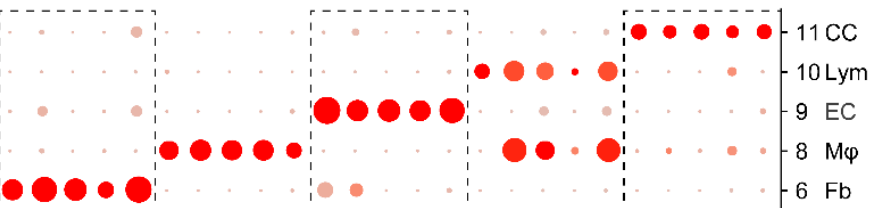

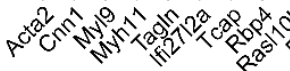

E
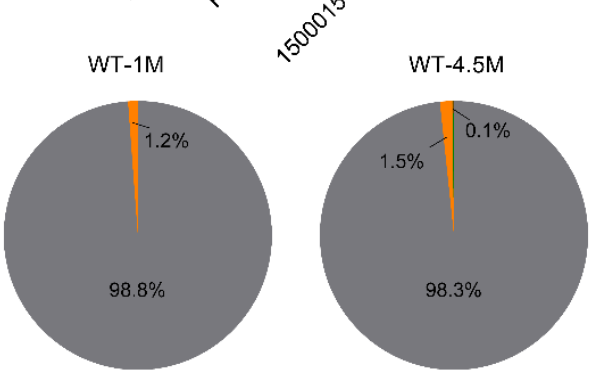

- VSMC

- Early modulated VSMC
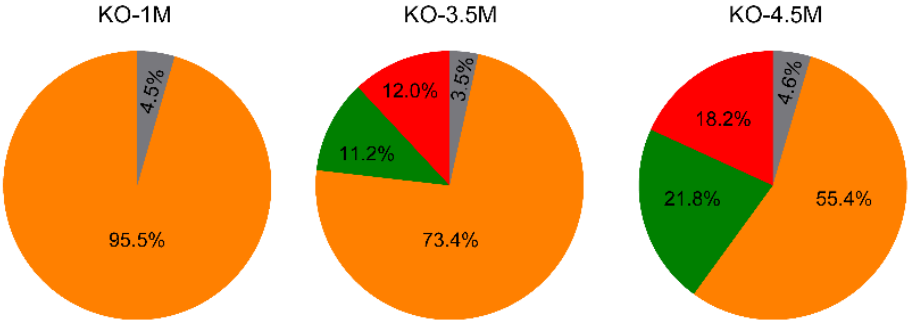

Fig.2. Single-cell RNA-seq identifies transformed cell populations in $\mathrm{Lkb1}^{\mathrm{SMiKo}}$ mice. (A)

Single-cell RNA-seq of the aortas from two independent samples of WT and $\mathrm{Lkbl}^{\text {SMiKO }}$ mice at 1.0 month (WT-1M and knockout [KO]-1M, respectively) post-tamoxifen (TAM) induction using 
the 10x Genomics Chromium platform. $t$-SNE clustering of 6928 single cells (3154 from WT-1M and 3774 from KO-1M) colored by sample origin, cell cluster, and cell type (VSMC, vascular smooth muscle cells; EM-VSMC, early modulated VSMCs; EC, endothelial cell; Fb, Fibroblast; $\mathrm{M} \varphi$, macrophage). (B) Single-cell RNA-seq of the aortas from three independent samples of WT and $L k b 1^{\text {SMiKO }}$ mice at 3.5 and 4.5 months post-TAM induction. $t$-SNE plots of 5560 single cells (1699 from WT-4.5M, 1642 from KO-3.5M, and 2219 from KO-4.5M), colored by sample origin, cell cluster, and cell type. Lym, lymphocyte; CC, cycling cell; Fb-like cell, fibroblast-like cell; Chon-like cell, chondrocyte-like cell. (C) Bar plot showing the relative distribution of each cluster identified in (B) in different samples of WT-4.5M, KO-3.5M, and KO-4.5M. (D) Bubble plot for gene expression of five cell-type differentiating markers. Dot color scale indicates the average expression level (Avg Exp) and dot size represents percentage of cells in each cluster that express at least one transcript of each gene (Pct Exp). (E) Pie chart showing percentage of each cell type among VSMCs of WT and $L k b 1^{S M i K O}$ mice at indicated time point post-TAM induction. 

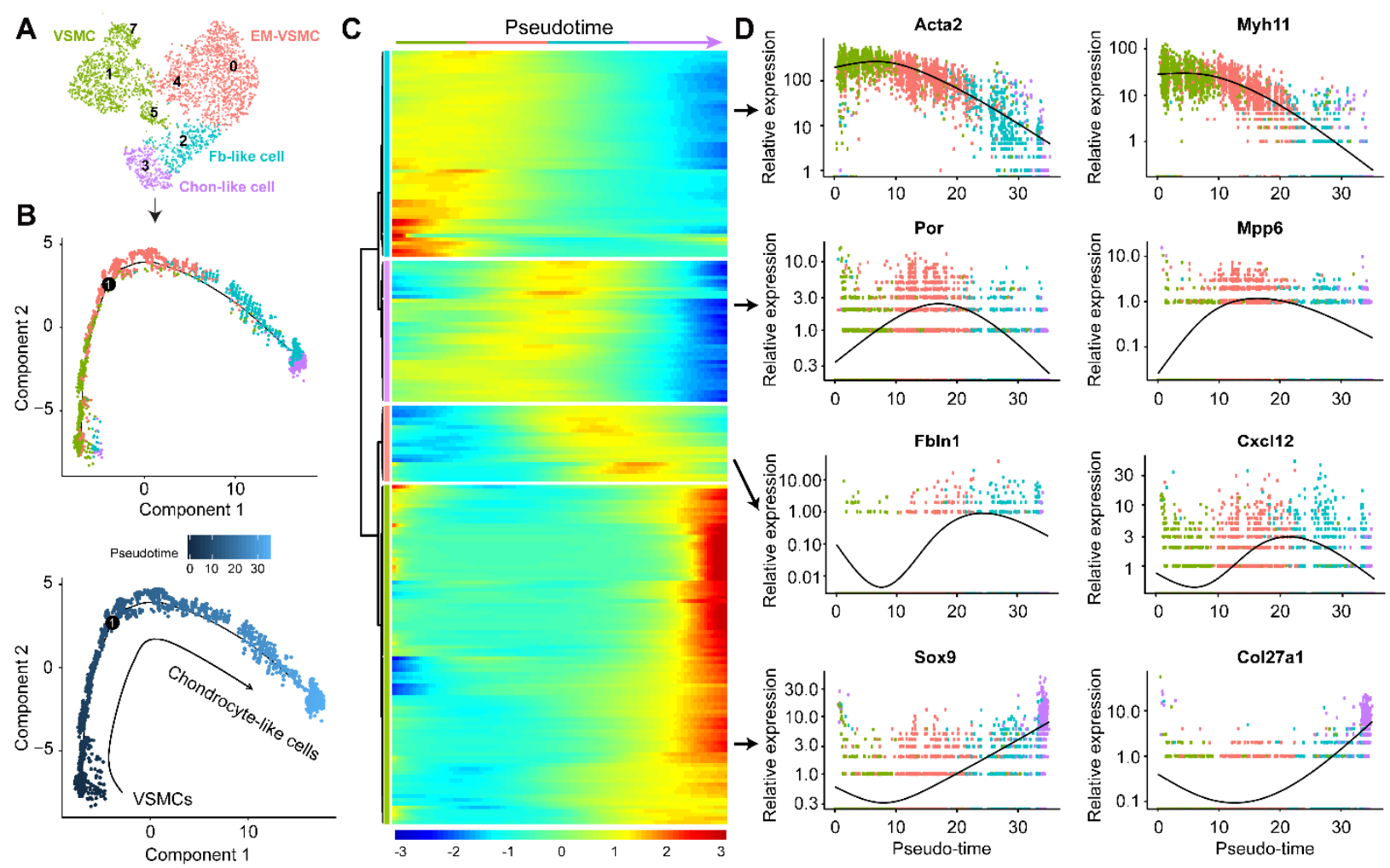

Fig.3. Single-cell trajectory reveals a VSMC transformation pathway, from early modulated VSMCs to fibroblast-like to chondrocyte-like cells in $\mathbf{L k b}^{\text {SMiKO }}$ mice. (A-B) Unsupervised analysis of single-cell gene expression profiles with Monocle revealed a linear trajectory. Cells within specific lineage clusters were selected, visualized using t-SNE visualization (Seurat $\mathrm{R}$ package), and then ordered based on a reversed graph embedding method (Monocle 2). (C) Heatmap of differentially expressed genes, ordered based on their common kinetics. The Expression $\mathrm{Z}$ score indicates changes in a gene relative to its dynamic range over pseudotime. (D) Kinetic diagrams showing the expression of some genes over pseudotime. 


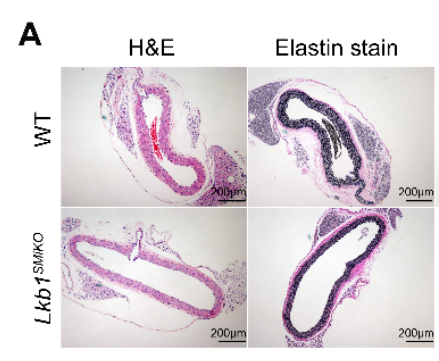

E
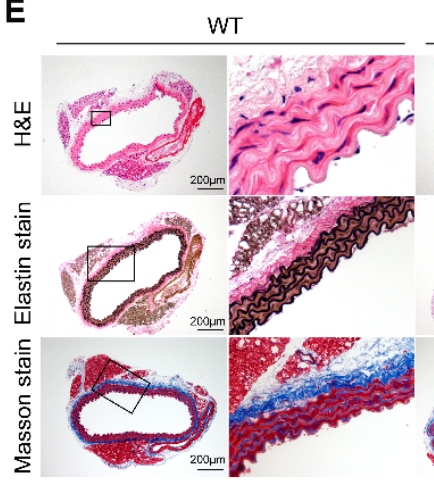

G
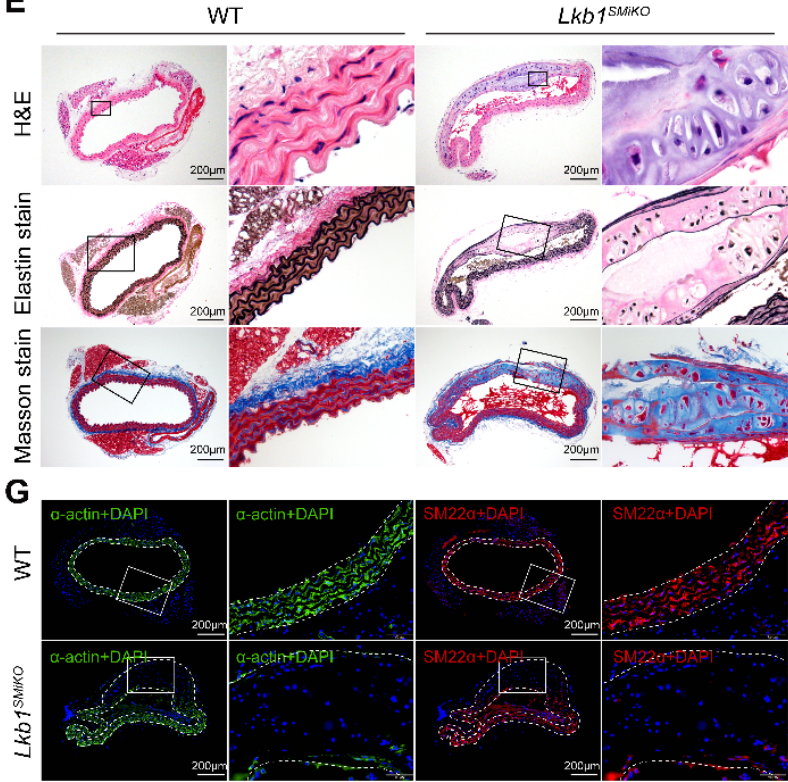

Lkb1 $1^{\text {SMiko }}$

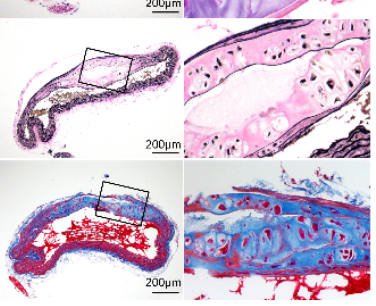

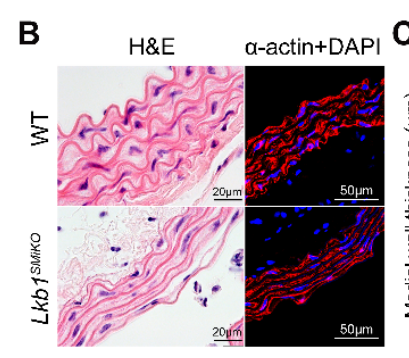
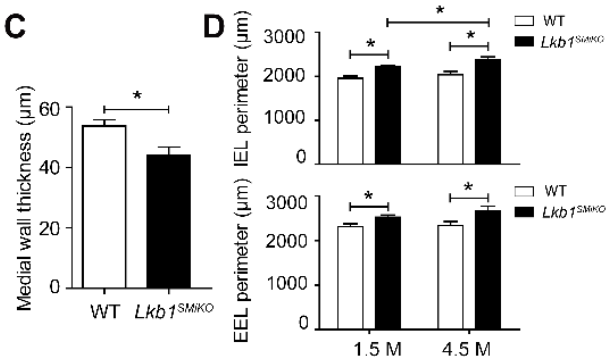

$\mathbf{F}$

WT Lkb1 $1^{\text {SMiko }}$

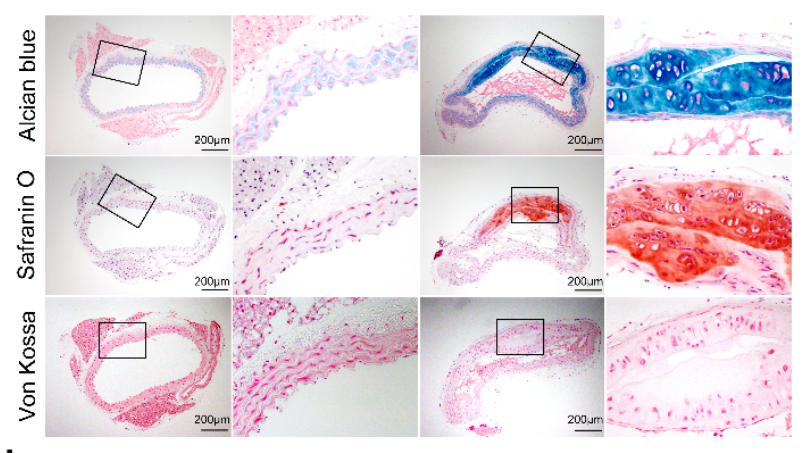

H

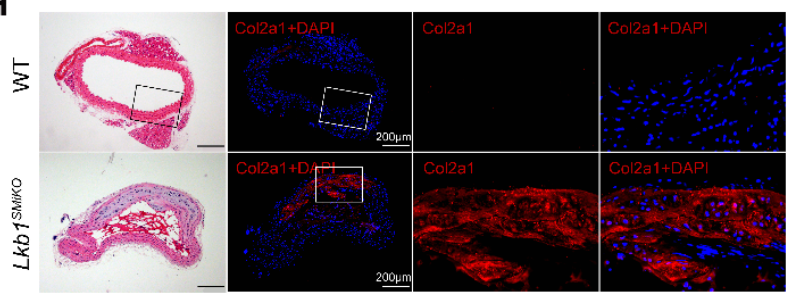

Fig.4. Histological analysis of the aortas from WT and $L \boldsymbol{k b} 1^{\text {SMiKo }}$ mice. (A) H\&E, elastin, and Masson trichrome stains of the thoracic aortas from WT and $\mathrm{Lkbl}^{\mathrm{SMiKO}}$ mice at $1.0-1.5$ months post-TAM induction. (B) H\&E staining and immunofluorescence staining ( $\alpha$-actin) of the thoracic aortas from WT and $\mathrm{Lkbl}^{\mathrm{SMiKO}}$ mice at 1.0-1.5 months post-TAM induction. (C) Quantification of the medial wall thickness of the thoracic aortas from WT and $L k b 1^{\text {SMiKO }}$ mice at 1.5 months post-TAM induction. (D) Quantification of the internal and external elastic laminae (IEL and EEL, respectively) perimeters of the thoracic aortas from WT and $L k b 1^{\text {SMiKO }}$ mice at 1.5 and 4.5 months post-TAM induction. (E) H\&E, elastin, and Masson stains of the thoracic aortas from WT and $\mathrm{Lkbl}^{\text {SMIKO }}$ mice at 4.5 months post-TAM induction. Boxes indicate fields shown at higher magnification in images to the immediate right. (F) Alcian blue, Safranin O, and Von Kossa staining of the thoracic aortas from WT and $\mathrm{Lkbl}^{\mathrm{SMiKO}}$ mice at 4.5 months post-TAM induction. (G and H) Immunofluorescence staining for $\alpha$-actin $(G), \operatorname{SM} 22 \alpha(G)$, and Col2a1 (H) of the thoracic aortas from WT and $L k b 1^{S M I K O}$ mice at 4.5 months post-TAM induction. Where indicated, nuclei were counterstained with DAPI. Dashed lines denote the IEL and EEL of the aortas. 


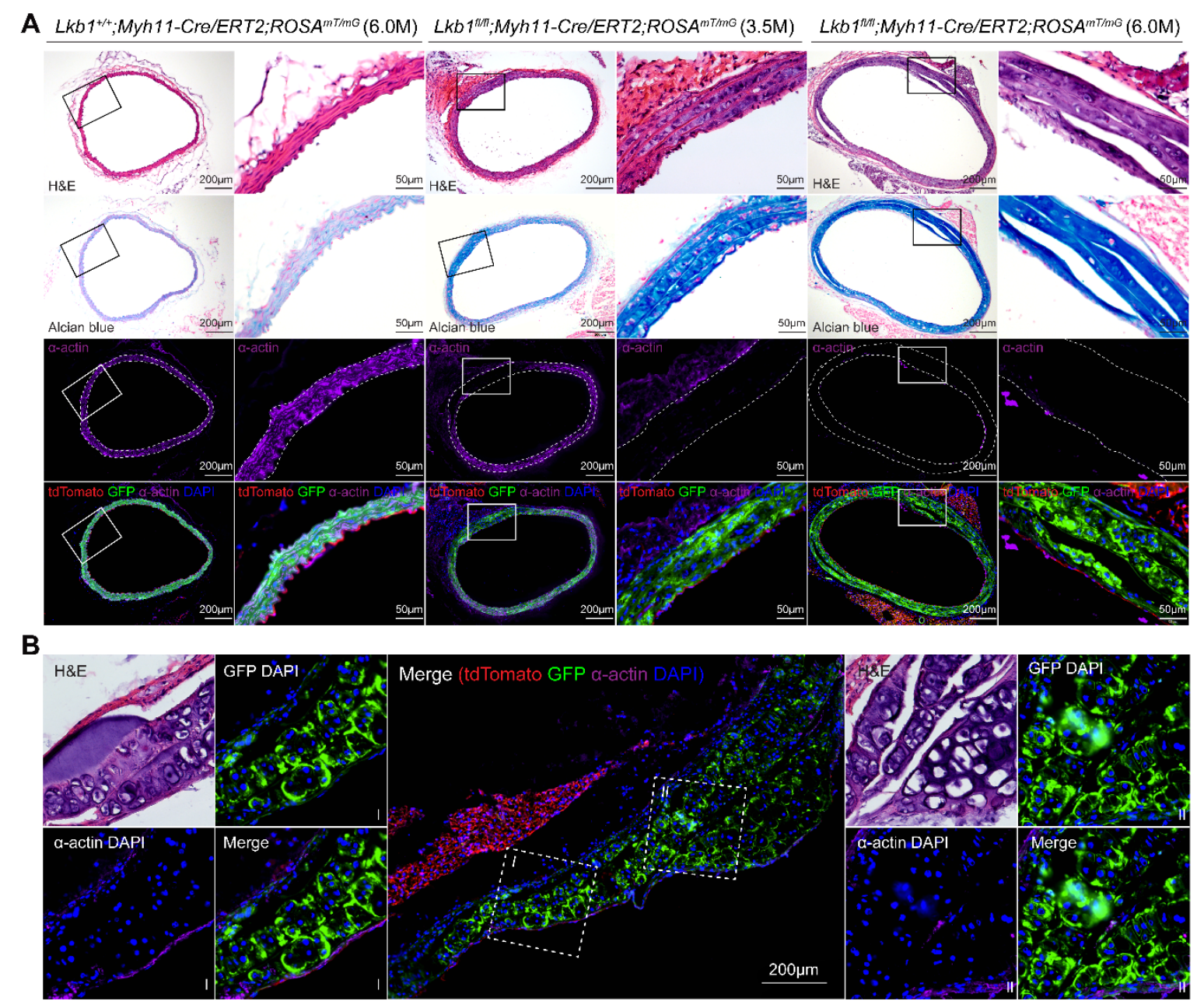

Fig. 5. Lineage tracing of VSMCs in $\mathbf{L k b 1}^{\text {SMiKo }}$ mice. (A) H\&E staining, Alcian blue staining, and anti- $\alpha$-actin co-immunostaining (purple) of thoracic aortas from $L k b 1^{+/+}$;Myhl1Cre/ERT2;ROSA ${ }^{m T /} m G$ and $L k b 1^{f l f l} ; M y h 11-C r e / E R T 2 ; R O S A^{m T / m G}$ mice at 3.5 and 6.0 months postTAM induction. Boxes indicate fields shown at higher magnification in images to the immediate right. Dashed lines denote the IEL and EEL of the aortas. (B) H\&E staining and anti- $\alpha$-actin coimmunostaining (purple) of aortic arch from Lkb1 $1^{f l / f l}$;Myh11-Cre/ERT2;ROSA ${ }^{m T / m G}$ mice at 3.5 months post-TAM induction. 

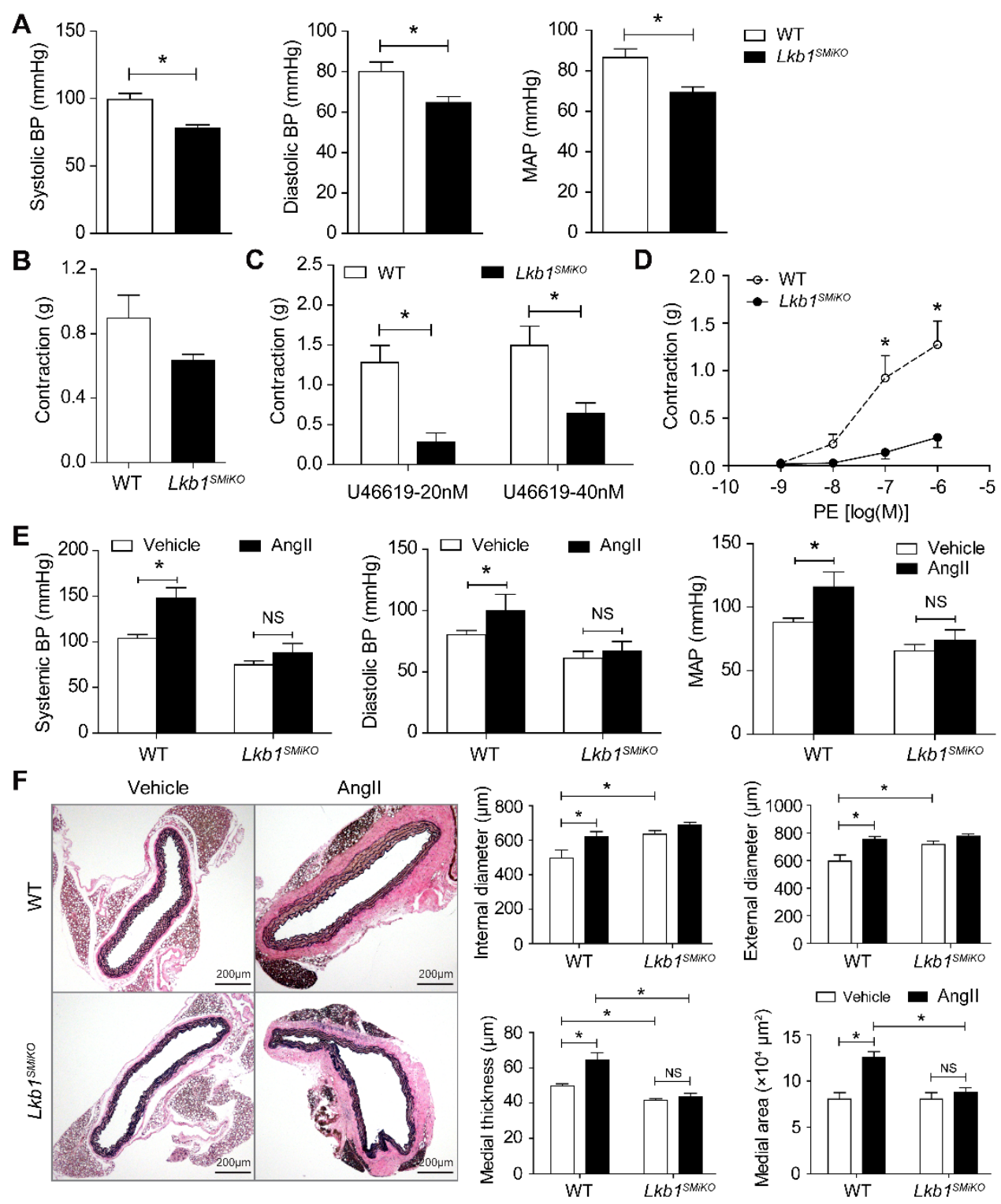

Fig. 6. Impaired vascular function in $\mathbf{L k b 1 ^ { S M i K o }}$ mice. (A) Blood pressure in WT and $L k b 1^{S M i K O}$ mice ( $n=4 /$ group). Blood pressure was measured by inserting fluid-filled catheters into carotid artery. ${ }^{*} \mathrm{p}<0.05$. (B-D) The first-order branches of the superior mesenteric arteries were isolated from WT and $\mathrm{Lkbl}^{\mathrm{SMiKO}}$ mice (two arteries for each mouse and five mice for each group). Vessel contraction induced by high (60 nM) potassium (B), U46619 (C), and phenylephrine (PE) (D) in control and $\mathrm{KO}$ mesenteric arteries was measured using wire myography. (E) Arterial blood pressure was measured by inserting fluid-filled catheters into the carotid artery in WT and LkbI ${ }^{\text {SMIKO }}$ mice infused with vehicle or angiotensin (Ang)II for 4 weeks. (F) Representative images of elastin staining and quantification of internal and external diameters, medial wall thickness, and 
bioRxiv preprint doi: https://doi.org/10.1101/2020.12.12.422410; this version posted December 12, 2020. The copyright holder for this preprint (which was not certified by peer review) is the author/funder. All rights reserved. No reuse allowed without permission.

medial area of the thoracic aortas from WT and $L k b 1^{S M i K O}$ mice infused with vehicle or AngII for 4 weeks. 